\title{
Control Algorithm Trajectory Planning for Dual Cooperative Manipulators with Experimental Verification
}

\author{
Ahmed Omar, Pan Ri and Yajun Zhang \\ College of Mechanical and Electrical Engineering, Beijing University of Chemical Technology, Beijing 100029, China
}

\begin{abstract}
In order to achieve cooperation of multi-robots simultaneously, this paper presents an approach of Trajectory planning for two decoupled robots with six degrees of freedom (DOF) each to execute packing task. The Denavit-Hartenberg (D-H) representation is used to model robot links and solve the transformation matrices of each joint. The inverse kinematics solution using for a 6- DOF Robotic arm is presented, for given initial and final configurations of the robots, the robots are placed so that their workspaces overlap. An algorithm successfully finds a set of configurations to ensure, collision free transition from start to end configuration. Simulation and experiments based on 6-dof robot are carried out and the results verified the effectiveness of the proposed algorithm.
\end{abstract}

\section{Introduction}

Dual arm manipulators demand has increased with the public expecting robots that mimic human's behavior. therefore, studying dual-arm manipulators is a natural avenue of researches [1]. variety of robot platforms used for dual arm manipulation, while some researches are carried out on systems built by simply placing two single arm manipulators for sharing same workspace [2].

The difficulty in mechanical analysis for dual arms comes mainly from the closed chain system, which is formed during the manipulation of the objects, and which is much more kinematically and dynamically complex than the typical serial manipulator configuration [3].

In general, control of multiple robot cooperation systems, the master-slave method is still the state of art for industrial robots [4]. The Master-Slave control strategy has to identify one robot as Master, the other as Slave.

A number of research works have been presented relating to the modelling of kinematics. Shi et al. have proposed the solution for forward kinematic problem of 6 DOF robot [5]. comprehensive survey can be found in [6].

In [7] analyzed the problem of path planning for two cooperating arm robots carrying an object between two given configurations, in a setup which includes obstacles. the method proposed extends the randomized potential field planning techniques introduced in [8].

The two keys contributions of this work are: (1) Designing control algorithm of the robots to conduct the prescribed task. (2) Cooperative robot manipulators system performing the assembly task. Suggested system is including two arm robots. Target of these robots is cooperating for Drug Packing.
This paper is structured as follows: firstly the proposed robotic platform and developed kinematic models introduced. Trajectory planning approach was discussed, then explains the design details of the electronic hardware. Finally Experimental results for two Cooperated robots are presented and concluded the work.

\section{Coordinate frames assignment}

The proposed system is containing two 6-DOF robots manipulators. This Robot has five rotational joints and a moving gripper. Figure 1 illustrates the graphical view of all the joints of the presented robot. Joint 1 represents the base and its axis of motion $z_{1}$. This joint provides a rotational $\theta_{1}$ angular motion around $\mathrm{z}_{1}$ axis in $\mathrm{x}_{1} \mathrm{y}_{1}$ plane. Joint 2 is identified as the Upper Arm and its axis is perpendicular to Joint 1 axis. It provides a rotational $\theta_{2}$ angular motion around $\mathrm{z}_{2}$ axis in $\mathrm{x}_{2} \mathrm{y}_{2}$ plane. $\mathrm{z}_{3}$ axes of Joint 3 and Joint 4 (Wrist) are parallel to Joint 2 z-axis; they provide $\theta_{3}$ and $\theta_{4}$ angular motions in $\mathrm{x}_{3} \mathrm{y}_{3}$ and $\mathrm{x}_{4} \mathrm{y}_{4}$ planes respectively.

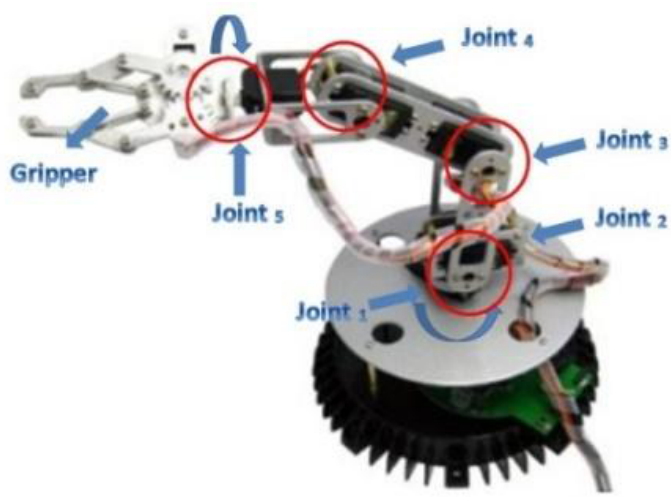




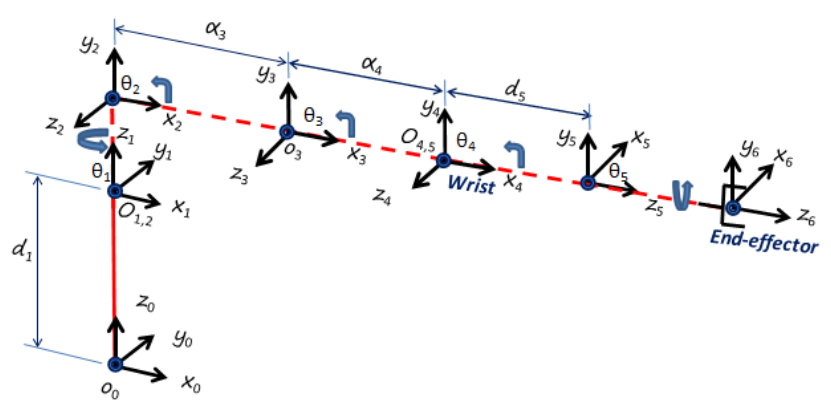

Figure 1. Coordinate robot frame.

\subsection{Denavit-Hartenberg (D-H) parameters}

The Denavit-Hartenberg analysis is one of the most used. depending on each mechanism. Based on the analysis, once one coordinate transformation between two frames is defined, D-H parameters for this robot defined for the assigned frames in Table 1.

Table 1. Link parameters of slave manipulators (D-H parameters)

\begin{tabular}{ccccc}
\hline No & $\boldsymbol{\alpha}_{\mathbf{i}-1 /\left({ }^{\circ}\right)}$ & $a_{\mathbf{i}-1 /(\mathbf{m m})}$ & $\mathbf{d}_{\mathbf{i} /(\mathbf{m m})}$ & $\boldsymbol{\theta}_{\mathbf{i}-1 /\left({ }^{\circ}\right)}$ \\
\hline 1 & 0 & 0 & 0 & $\theta_{1}$ \\
2 & 90 & 0 & 93 & $\theta_{2}$ \\
3 & 0 & 80 & 0 & $\theta_{3}$ \\
4 & 0 & 81 & 0 & $\theta_{4}$ \\
5 & -90 & 0 & 22 & $\theta_{5}$ \\
6 & 0 & 0 & 0 & Gripper \\
\hline
\end{tabular}

Based on these parameters, the transformation matrix includes the overall rotation and translation of tool frame $\{\mathrm{B}\}$ with respect to base frame $\{\mathrm{A}\}$.

Assume that, $a_{i}$ is the length of Manipulator's link i, $\theta_{i}$ is the angle of joint i, $\alpha_{i}$ is the angle between link i and link $\mathrm{i}-1, \mathrm{~d}_{\mathrm{i}}$ the distance between link $\mathrm{i}$ and link $\mathrm{i}-1$, where $\mathrm{i}=1,2, \ldots, 6 . a_{i}, \alpha_{i}, d_{i}$ are all known and $\theta_{i}$ is proposed to be obtained In this paper, the link parameters of robot are given in Table 1 above.

According to calculated ${ }_{\mathrm{P}_{2}}^{\mathrm{O}_{2}} \boldsymbol{T}$, suppose that:

$$
\begin{gathered}
\theta_{1}=\arctan 2(x, y) \\
\theta_{2}=\arctan 2\left(s_{2}, c_{2}\right) \\
\theta_{3}=\arctan 2\left(s_{3}, c_{3}\right) \\
\theta_{234}=\arctan 2\left(s_{234}, c_{234}\right) \\
\theta_{4}=\arctan 2\left(s_{4}, c_{4}\right) \\
\theta_{5}=\arctan 2\left(s_{5}, c_{5}\right)
\end{gathered}
$$

\section{Analysis and calculation of dual robots}

In space geometry, using a unique $4 \times 4$ homogeneous transformation matrix ${ }_{\mathrm{A}}^{\mathrm{B}} \boldsymbol{P}$ can describe the motion from coordinate system $\{\mathrm{B}\}$ of Point $\mathrm{B}$ to coordinate system $\{$ A $\}$ of Point A, i.e:

$$
{ }_{\mathrm{A}}^{\mathrm{B}} \boldsymbol{P}=\left[\begin{array}{cc}
{ }_{\mathrm{A}}^{\mathrm{B}} \boldsymbol{R} & { }_{\mathrm{A}}^{\mathrm{B}} \boldsymbol{T} \\
\boldsymbol{O} & 1
\end{array}\right]
$$

where ${ }_{\mathrm{A}}^{\mathrm{B}} \boldsymbol{R} \in \mathbf{R}^{3 \times 3}$ is rotation matrix, ${ }_{\mathrm{A}}^{\mathrm{B}} \boldsymbol{T} \in \mathbf{R}^{3 \times 3}$ is translation matrix. If a series of motions appear, composite transformation will be a good choice to describe.

Assume that, $\left\{\mathrm{O}_{1}\right\}\left(\left\{\mathrm{O}_{2}\right\}\right)$ is the base coordinate system of master (slave) manipulator and $\left\{\mathrm{P}_{1}\right\} \quad\left(\left\{\mathrm{P}_{2}\right\}\right)$ is the end coordinate system of master (slave) manipulator as shown in Fig. 2. Then ${ }_{\mathrm{P}_{1}}^{\mathrm{O}_{1}} \boldsymbol{T}\left({ }_{\mathrm{P}_{2}}^{\mathrm{O}_{2}} \boldsymbol{T}\right)$ is a transformation matrix from the end coordinate system to the base coordinate system of master (slave) manipulator.

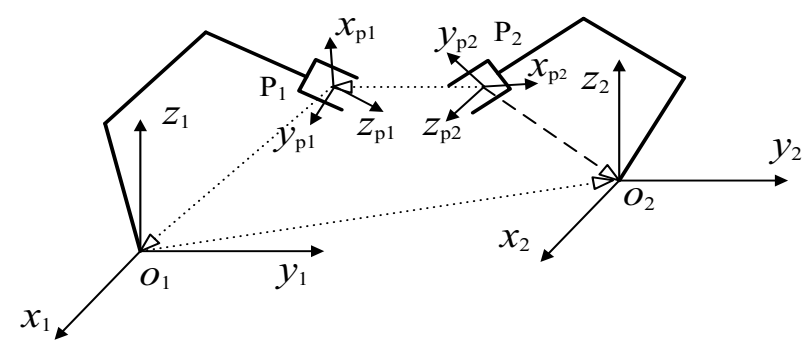

Figure 2. The simplified model of dual manipulators cooperating at $t_{0}$.

${ }_{\mathrm{P}_{2}}^{\mathrm{O}_{2}} \boldsymbol{T}$ could be calculated through composite transformation. It is a transformation from $\left\{\mathrm{P}_{2}\right\}$ to $\left\{\mathrm{P}_{1}\right\}$, then from $\left\{\mathrm{P}_{1}\right\}$ to $\left\{\mathrm{O}_{1}\right\}$, and from $\left\{\mathrm{O}_{1}\right\}$ to $\left\{\mathrm{O}_{2}\right\}$. Complying with the rules of the rotation matrix multiplication, we obtain ${ }_{\mathrm{P}_{2}}^{\mathrm{O}_{2}} \boldsymbol{T}$ as:

$$
{ }_{\mathrm{P}_{2}}^{\mathrm{O}_{2}} \boldsymbol{T}={ }_{\mathrm{O}_{1}}^{\mathrm{O}_{2}} \boldsymbol{T} \cdot{ }_{\mathrm{P}_{1}}^{\mathrm{O}_{1}} \boldsymbol{T} \cdot{ }_{\mathrm{P}_{2}}^{\mathrm{P}_{1}} \boldsymbol{T}
$$

${ }_{\mathrm{O}_{1}}^{\mathrm{O}_{2}} \boldsymbol{T}$ is known because two bases of manipulators must be fixed before they work. After the master manipulator teaching, ${ }_{\mathrm{P}_{1}}^{\mathrm{O}} \boldsymbol{T}$ is obtained. According to given tasks, we can obtain ${ }_{\mathrm{P}_{2}}^{\mathrm{P}_{1}} \boldsymbol{T}$. So the transformation ${ }_{\mathrm{P}_{2}}^{\mathrm{O}_{2}} \boldsymbol{T}$ can be obtained in the case where ${ }_{\mathrm{O}_{1}}^{\mathrm{O}_{2}} \boldsymbol{T},{ }_{\mathrm{P}_{1}}^{\mathrm{O}_{1}} \boldsymbol{T},{ }_{\mathrm{P}_{2}}^{\mathrm{P}_{2}} \boldsymbol{T}$ are known. Therefore, the transformation matrix ${ }_{\mathrm{P}_{2}}^{\mathrm{O}_{2}} \boldsymbol{T}$ at any time $t$ can be written as:

$$
{ }_{\mathrm{P}_{2}}^{\mathrm{O}_{2}} \boldsymbol{T}={ }_{\mathrm{O}_{1}}^{\mathrm{O}_{2}} \boldsymbol{T} \cdot{ }_{\mathrm{P}_{1}}^{\mathrm{O}_{1}} \boldsymbol{T}(t) \cdot{ }_{\mathrm{P}_{2}}^{\mathrm{P}_{1}} \boldsymbol{T}_{(t)}
$$

If the dual-manipulator collaborative movement is coupled motion, ${ }_{\mathrm{P}_{2}}^{\mathrm{P}_{(t)}} \boldsymbol{T}_{(\mathrm{s}}$ a constant matrix because the 
relative relation of the extremity pose between master manipulator and slave manipulator during their movements is constant. It is particular case of dual manipulators collaborative movements and can be described as:

$$
{ }_{\mathrm{P}_{2}}^{\mathrm{O}_{2}} \boldsymbol{T}={ }_{\mathrm{O}_{1}}^{\mathrm{O}_{2}} \boldsymbol{T} \cdot{ }_{\mathrm{P}_{1}}^{\mathrm{O}_{1}} \boldsymbol{T}(t) \cdot{ }_{\mathrm{P}_{2}}^{\mathrm{P}_{2}} \boldsymbol{T}
$$

\section{Trajectory planning}

Trajectory planning for robotic manipulators is the process of creating trajectories free of collisions allowing the manipulators to perform the required task. the trajectory calculation formulas of Dual Manipulators under two different collaborative movements were put forward in literature (10). trajectory planning of Dual Manipulators is transformed into the teaching trajectory of single Manipulator through calculation. However, the algorithm given in the literature (10) is complicated. Different formulas are bound to be used in different circumstances.

\subsection{Transformation from initial to final point}

In this experiment work Master Manipulator was required to move from initial position $\mathrm{A}_{1}[0,0,0]$ to a given position $\mathrm{B}_{1}[0,290,20]$ to pick up the Bottle, then to position $C_{1}[0,250,170]$ to position $D_{1}[250,0,170]$ in the Cooperated area and finally to initial position again $\mathrm{A}_{1}[0,0,0]$ in 12 Seconds. And the Slave Manipulator was required to move from initial position $\mathrm{A}_{2}[0,0,0]$ to a given position $\mathrm{B}_{2}[0,290,20]$ to pick up the $\mathrm{Box}$, then to position $C_{2}[0,250,100]$ to position $D_{2}[-250,0,100]$ in the Cooperated area then to position $\mathrm{E}_{2}[-210,210,100]$ to locate it in the Delivery area and finally to initial position again $\mathrm{A}_{2}[0,0,0]$ in 14 Seconds. Figure 3 illustrates the top view of packing process task.

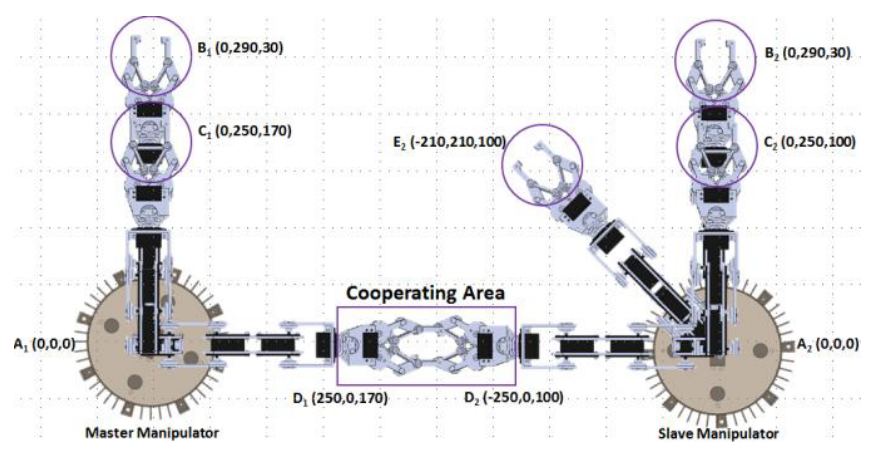

Figure 3. Cooperation process (master $\&$ slave) manipulators.

The motion was separated to four segments for Master Manipulator and five segments for Slave Manipulator, defined by the time period .Figure 4-5 illustrate the angle Values of the Joints of Master and Slave Manipulator in whole motion segments, the initial position $(0,0,0)$ will be used as a reference to all joint angles of the robots.
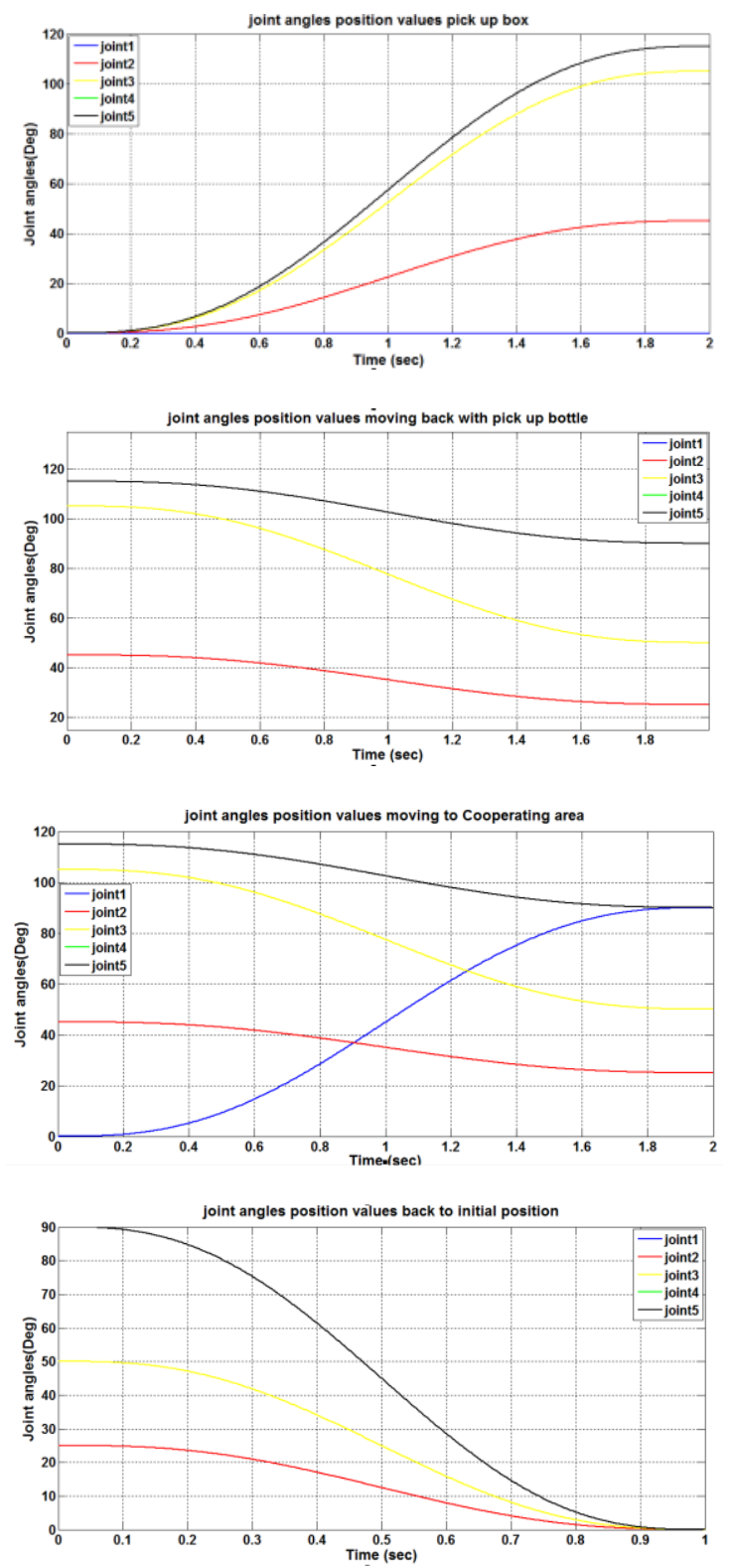

Figure 4. Angle values of master manipulator joints.
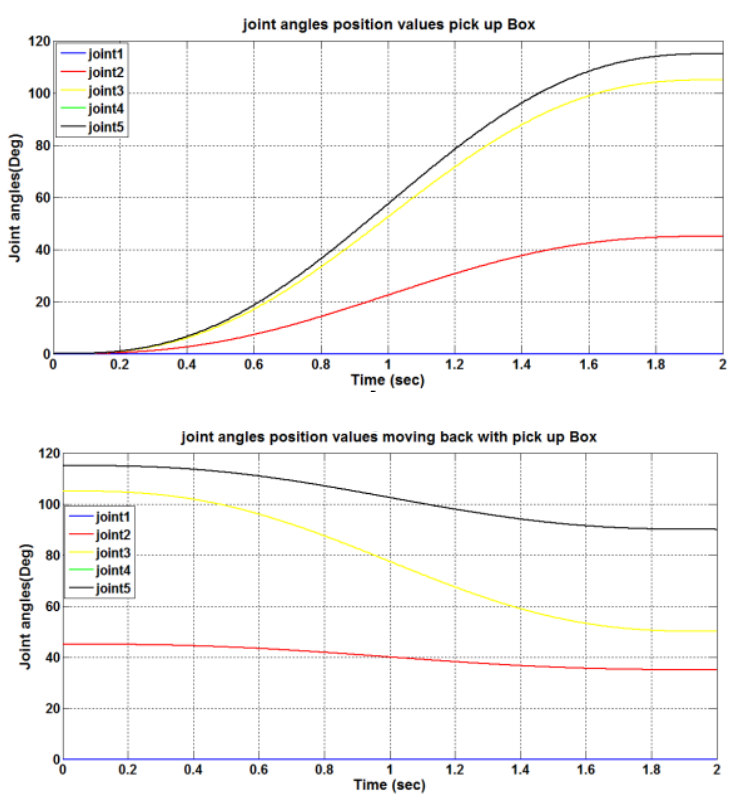

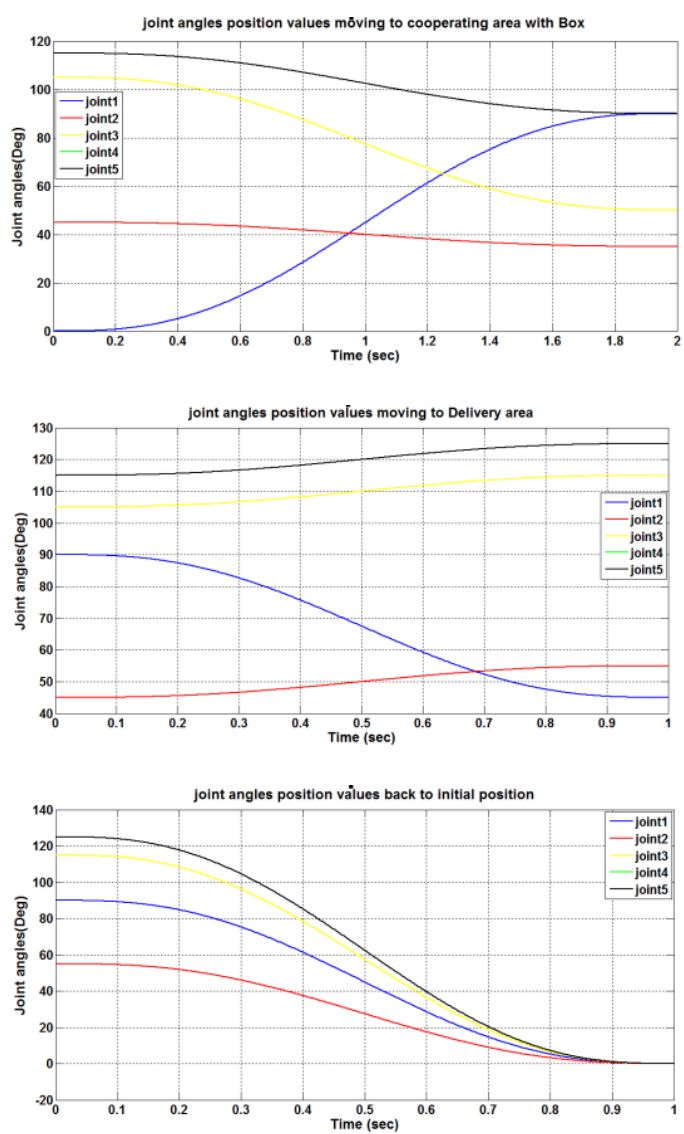

Figure 5. Angle values of slave manipulator joints.

\section{Experimental platform}

Double Manipulators, Arduino controller board, PC, 2 infrared distance sensors, 2 external power supplies $(5 \mathrm{~V} / 1 \mathrm{~A} \& 6 \mathrm{~V} / 3 \mathrm{~A})$ are needed in this experiment. Arduino controller board with Atmegal 168. The sensors GP2Y0A21 can measure the distance of less than $80 \mathrm{~cm}$.

\section{Experimental results and discussion}

Experiments of Dual arm Robots in Cooperating task motion are carried out. As shown in Figure 6, Master robot picks a bottle while Slave carries a Drug box. The slave robot tracks the trajectories on the box while the master transports the bottle. sensory feedback used to add an advantage of closed loop controller for the control system.

Execute the program and perform operation, the whole process will be as follows:

Master and slave Manipulators were moved from initial to final positions as discussed previously. whole real sequence process of Dual Manipulators cooperation for drug Packing shown in Figure 6.

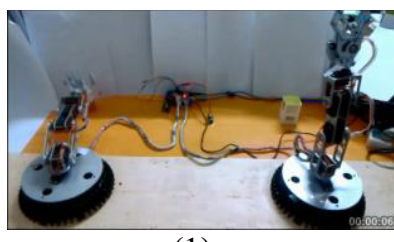

(1)

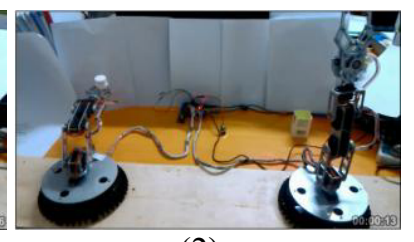

(2)

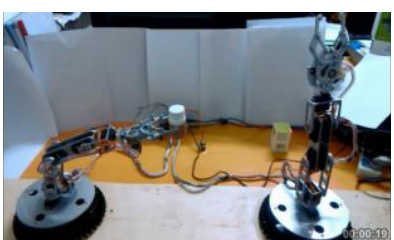

(3)

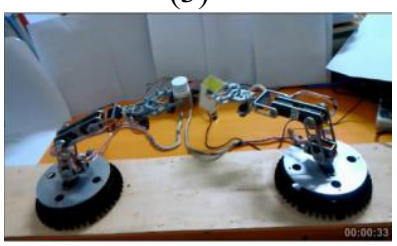

(5)

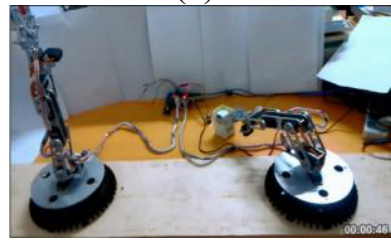

(7)

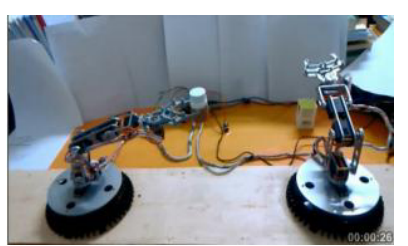

(4)

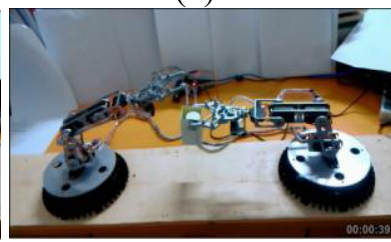

(6)

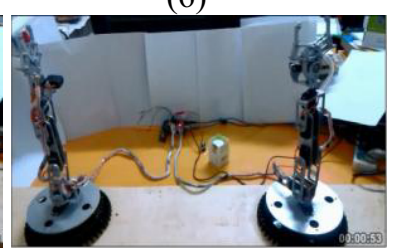

(8)
Figure 6. Cooperation process task of dual manipulators.

In this experiment, the control system was set up and the task of dual manipulators cooperating for drug packing was performed using the method put forward before. By the above figures, it is clear that the two robots can move in a Cooperative motion Therefore, the results verified the effectiveness of the proposed algorithm.

\section{Conclusion}

The aims of this paper were to establish the theoretical foundation for automated cooperative trajectory planning of Dual arm robots. Motion constraints between cooperative master and slave robots are the basis for this method. Denavit-Hartenberg (D-H) representation is used to model robot links and solve the transformation matrices of each joint. The inverse kinematics solution for a 6- DOF Robotic arm was presented. the proposed algorithm was successfully implemented on the packing Cooperating task. this work was motivated bearing in mind that the two arm robots could be controlled by one controller simultaneously in centralized fashion that would reduce the cost of production effectively.

\section{Acknowledgement}

This work was financially supported by the Fundamental Research funds for the Central Universities (YS1403, ZY1521).

\section{References}

1. Ch. Smith, Y. Karayiannidis, L. Nalpantidis, X. Gratal, P. Qi, D. V. Dimarogonas, D. Kragic, Dual arm manipulation - A survey, Robotics and Autonomous. 
2. F. Caccavale, P. Chiacchio, A. Marino, L.Villani,impedance control of dual-arm cooperative manipulators, IEEE/ASME Transactions on Mechatronics 13, 5576586 (2008)

3. S. Al-Yahmadi, J. Abdo and T. C. Hsia, Modeling and control of two manipulators handling a flexible object, Journal of the Franklin Institute, 344 349-361 (2007)

4. KUKA Roboter GmbH. KUKA.CR Issued: 21 Aug 2007. Motion Cooperation 2.1 For KUKA System Software (KSS) 5.3/5.4/5.5, Version: 00.

5. X. Shi and N. G. Fenton, A complete and general solution to the forward kinematics problem of platform-type robotic manipulators, IEEE International Conference on Robotics and Automation Proceedings, 3055-3062 (1994)

6. Latombe, J. C. Robot Motion Planning. Kluwer Academic Publishers, Boston (1991)

7. Y. Koga, J.-C. Latombe, Experiments in dual-arm manipulation planning, in: IEEE International Conference on Robotics and Automation, 3, 22382245 (May 1992)

8. J. Barraqu and J. C. Latombe, Robot motion planning: a distributed representation approach, Stanford University, (May 1989) 